\title{
Origins of Staphylococcus epidermidis and Streptococcus oralis causing bacteraemia in a bone marrow transplant patient
}

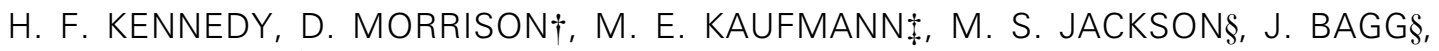 \\ B. E. S. GIBSON*, C. G. GEMMELL† and J. R. MICHIE
}

Departments of Microbiology and *Haematology, Royal Hospital for Sick Children, Yorkhill NHS Trust, Glasgow, †Scottish MRSA Reference Laboratory and University Department of Bacteriology, Glasgow Royal Infirmary, Glasgow, \$Epidemiological Typing Unit, Laboratory of Hospital Infection, Central Public Health Laboratory, London and §Department of Oral Microbiology, Glasgow Dental Hospital and School, Glasgow

\begin{abstract}
Coagulase-negative staphylococcal bacteraemia in immunocompromised patients is often associated with the use of central venous catheters, while the proposed origin of viridans streptococci causing bacteraemia in this patient group is the oral cavity. This report describes an episode of polymicrobial bacteraemia caused by Staphylococcus epidermidis and Streptococcus oralis followed by several further episodes of $S$. epidermidis bacteraemia in a 15-year-old boy after bone marrow transplantation. Pulsed-field gel electrophoresis (PFGE) of Sma I chromosomal DNA digests was used to compare blood culture and oral isolates of $S$. epidermidis and Str. oralis. The results indicated that the mouth was the source of both $S$. epidermidis and Str. oralis causing the first episode of bacteraemia. PFGE further demonstrated that the central venous catheter was the origin of a second strain of $S$. epidermidis responsible for subsequent episodes of staphylococcal bacteraemia. Both the oral mucosa and central venous lines should be considered as potential sources of organisms, including coagulase-negative staphylococci, associated with bacteraemia in immunocompromised patients.
\end{abstract}

\section{Introduction}

During the first 4 weeks following allogeneic bone marrow transplantation, recipients are particularly susceptible to bacterial infection because of profound neutropenia resulting from their conditioning regimen. Concommitantly, severe oral mucositis allows endogenous bacteria to gain access to the bloodstream, while supportive care - such as the use of central venous catheters - provides further portals of entry for development of systemic and local infection.

Coagulase-negative staphylococci (CNS) have become the most frequently isolated bacteria from blood cultures of febrile neutropenic patients. These organisms are skin commensals, regarded as opportunist pathogens, particularly in association with the use of intravenous catheters $[1,2]$. CNS adhere to the catheter polymer and produce an extracellular slime substance

Received 4 Aug. 1999; accepted 9 Sept. 1999.

Corresponding author: Dr H. F. Kennedy. which forms a biofilm protecting bacteria from antibiotic activity.

Viridans streptococci comprise a significant part of the normal flora of the oral cavity and previously had few disease associations other than dental caries and, much less commonly, endocarditis. However, over the last decade these organisms have become important pathogens in neutropenic patients. Viridans streptococcal bacteraemia may have a variable course, including a severe form characterised by persistent fever, acute respiratory distress and in some instances septic shock [3,4]. Richards et al. [5] used ribotyping to demonstrate that the mouth can be the source of these organisms, which gain access to the bloodstream via chemotherapy-induced oral mucositis.

This paper describes coagulase-negative staphylococcal and viridans streptococcal bacteraemia in a bone marrow transplant patient. To determine the source of organisms causing bacteraemia, the biotype, antibiogram and PFGE type of each isolate from blood cultures were compared with isolates of coagulase- 
negative staphylococci and viridans streptococci colonising the patient.

\section{Case report}

A 15-year-old boy with Philadelphia positive, chronic myeloid leukaemia was treated with hydroxyurea from diagnosis, followed by bone marrow transplantation from a matched unrelated donor at 9 months from presentation. Conditioning consisted of cyclophosphamide, total body irradiation and Campath IG. Graftversus-host disease prophylaxis consisted of cyclosporin and methotrexate.

On day 7 post-transplant, while neutropenic and with severe oral mucositis, the patient became febrile. Viridans streptococci and CNS were isolated from blood culture. Vancomycin was added to first-line antimicrobial therapy of amikacin plus piperacillin/tazobactam. On day 11 , CNS were again cultured from blood. By day 21 the patient was apyrexial and repeat blood cultures were negative.

Throughout the period of neutropenia (until day 23), weekly surveillance cultures (days 4, 11 and 21) yielded CNS from all mouth swabs and from one nose swab (day 11). Surveillance cultures of the Hickman line exit site, faeces and urine yielded no evidence of CNS. After neutrophil recovery, cultures from the same sites on day 25 yielded CNS from the mouth swab only. Oral mucositis was most severe between days 5 and 9 and did not resolve completely until day 24 .

Two more episodes of CNS bacteraemia on days 29 and 70 responded rapidly to vancomycin therapy, which allowed the Hickman line to be retained in situ. However, following a more acute episode of septicaemia with CNS, beginning on day 150, the line was removed (day 153). Echocardiography performed at regular intervals was negative throughout. After removal of the Hickman line, CNS bacteraemia did not recur.

\section{Materials and methods}

\section{Identification of bacteria and antibiotic susceptibility testing}

All infecting or colonising isolates of staphylococci and streptococci were collected and identified by the API STAPH or Rapid ID 32 STREP method (bioMérieux, Basingstoke), respectively. All antibiotic susceptibility patterns except that of methicillin were determined for each isolate with sensitivity disks according to the method of Stokes [6] on Diagnostic Sensitivity Agar with lysed horse blood (E \& O Laboratories, Bonnybridge) 5\%. Staphylococcus aureus NCTC 6571 was used as the control organism. Plates were incubated for $18 \mathrm{~h}$ at $37^{\circ} \mathrm{C}$. Antibiotics tested were $(\mu \mathrm{g})$ vancomycin
(30), rifampicin (2), clindamycin (2), fusidic acid (10), ciprofloxacin (1), amikacin (30) and erythromycin (5). Methicillin susceptibility was tested with $25-\mu \mathrm{g}$ strips on Columbia blood agar plates incubated overnight at $30^{\circ} \mathrm{C}$.

\section{Pulsed-field gel electrophoresis (PFGE)}

PFGE was performed on all isolates of coagulasenegative staphylococci as all possessed the same biochemical identification profile. One isolate of viridans streptococcus from the mouth and the strain from blood culture with identical biochemical identification profiles were also compared by PFGE.

Cultures were grown overnight in brain heart infusion broth, $0.5 \mathrm{ml}$ was washed in NET buffer $(10 \mathrm{mM}$ Tris, $1 \mathrm{mM}$ EDTA, $10 \mathrm{mM} \mathrm{NaCl}$ ) and resuspended in $0.25 \mathrm{ml}$ of NET buffer. Lysozyme (1 mg), mutanolysin (100 units) and RNAase $(25 \mu \mathrm{g})$ were added to the cell suspension and mixed with an equal volume of SeaPlaque GTG agarose (Flowgen) $2 \%$ at $50^{\circ} \mathrm{C}$. The cell/agarose suspension was pipetted into a block mould and allowed to solidify at $4^{\circ} \mathrm{C}$. Cells were lysed at $37^{\circ} \mathrm{C}$ for $2-3 \mathrm{~h}$ in lysis buffer (lysozyme $1 \mathrm{mg} / \mathrm{ml}$, RNAase $25 \mu \mathrm{g} / \mathrm{ml}, 6 \mathrm{mM}$ Trizma base, $100 \mathrm{~mm}$ EDTA, $1 \mathrm{M} \mathrm{NaCl}$, Brij $580.5 \%$, sodium deoxycholate $0.2 \%$, lauroyl sarcosine $0.5 \%$ ) followed by a further overnight incubation at $50^{\circ} \mathrm{C}$ in proteolysis buffer (proteinase $\mathrm{K}$ $100 \mu \mathrm{g} / \mathrm{ml}$, lauroyl sarcosine $1 \%$ in $0.5 \mathrm{M}$ EDTA). The blocks were washed three times for 10 min each in TE buffer (10 mM Trizma base, $1 \mathrm{~mm}$ EDTA). The DNA was digested with Sma I (30 units) for $2-3 \mathrm{~h}$ according to the manufacturer's instructions and electrophoresed in an agarose (Molecular Grade Agarose, BioRad Laboratories) $1 \%$ gel in $0.5 \times \mathrm{TBE}$ buffer $(44.5 \mathrm{mM}$ Trizma base, $44.5 \mathrm{~mm}$ boric acid, $1 \mathrm{mM}$ EDTA) by the contour-clamped homogeneous electric field (CHEF) method with a CHEF-Mapper drive module (BioRad Laboratories). The gels were run for $23 \mathrm{~h}$ with a linear ramped pulse time of $6.75-63.8 \mathrm{~s}$ and stained with ethidium bromide $1 \mu \mathrm{g} / \mathrm{ml}$ for $30 \mathrm{~min}$. Chemicals were purchased from Sigma UK unless otherwise indicated.

\section{Results}

All staphylococci were identified as $S$. epidermidis (with the same biochemical identification profile). The viridans streptococcal isolate from the first positive blood culture (day 7) and two others from the mouth swab of day 4 were identified as Str. oralis. The biochemical identification profile of the Str. oralis from blood was identical to that of one oral isolate.

Antibiotic susceptibility tests suggested the presence of two strains of $S$. epidermidis. All isolates were susceptible to vancomycin, rifampicin and clindamycin and resistant to methicillin, ciprofloxacin and erythromycin (Table 1). Six isolates (Table 1, antibiogram A) 
Table 1. Antibiotic susceptibility and PFGE analysis of the $S$. epidermidis isolates

\begin{tabular}{rlcc}
\hline $\begin{array}{l}\text { Days after } \\
\text { transplant }\end{array}$ & Site & Antibiogram* & PFGE type \\
\hline 4 & Mouth & A & 1 \\
7 & Blood & A & 1 \\
11 & Blood & B & 2 \\
11 & Mouth & A & 1 \\
21 & Mouth & A & 1 \\
21 & Nose & A & 1 \\
25 & Mouth & A & 1 \\
29 & Blood & B & 2 \\
70 & Blood & B & 2 \\
150 & Blood & B & 2 \\
153 & Line tip & B & 2 \\
\hline
\end{tabular}

*Antibiogram A: susceptible to vancomycin, rifampicin and clindamycin and resistant to methicillin, fusidic acid, ciprofloxacin, erythromycin and amikacin. Antibiogram B: susceptible to vancomycin, rifampicin, clindamycin, fusidic acid, amikacin and resistant to methicillin, ciprofloxacin and erythromycin.

were also resistant to fusidic acid and amikacin while five were susceptible (Table 1, antibiogram B).

PFGE of Sma I chromosomal DNA digests of the $S$. epidermidis isolates divided them into two distinct pattern types (pulsed-field types 1 and 2), which differed from each other by $>10$ bands (Fig. 1). The pulsed-field types corresponded exactly to the two different antibiograms (Table 1). The isolate of $S$. epidermidis recovered from the first positive blood culture (day 7) and all isolates from positive surveillance cultures (mouth swabs taken on days 4, 11, 21, 25 and one nose swab taken on day 11) belonged to PFGE type 1, while subsequent blood culture isolates (days 11, 29, 70, 150) and the $S$. epidermidis from the tip of the Hickman line (day 153) belonged to PFGE type 2.

The isolates of Str. oralis from blood and mouth, with identical biochemical identification profiles and antibiograms, were also identical by PFGE (Fig. 1).

\section{Discussion}

These results suggest that during a period of neutropenia with severe mucositis, the patient's mouth was the source of both Str. oralis and S. epidermidis PFGE type 1 isolates responsible for the first episode of bacteraemia. Although mucositis persisted for a further 17 days, subsequent episodes of bacteraemia were caused by $S$. epidermidis PFGE type 2, the source of which was the tip of the patient's Hickman line. All surveillance cultures of the mouth demonstrated continued colonisation with $S$. epidermidis PFGE type 1 for several weeks. The very severe nature of mucositis during the first 10 days after bone marrow transplantation may have provided the greatest opportunity for $S$. epidermidis PFGE type 1 to gain access to the bloodstream. The oral origin of this strain was also supported by the concurrent isolation of identical strains of Str. oralis (by API type, antibiogram and PFGE) from both mouth and blood.

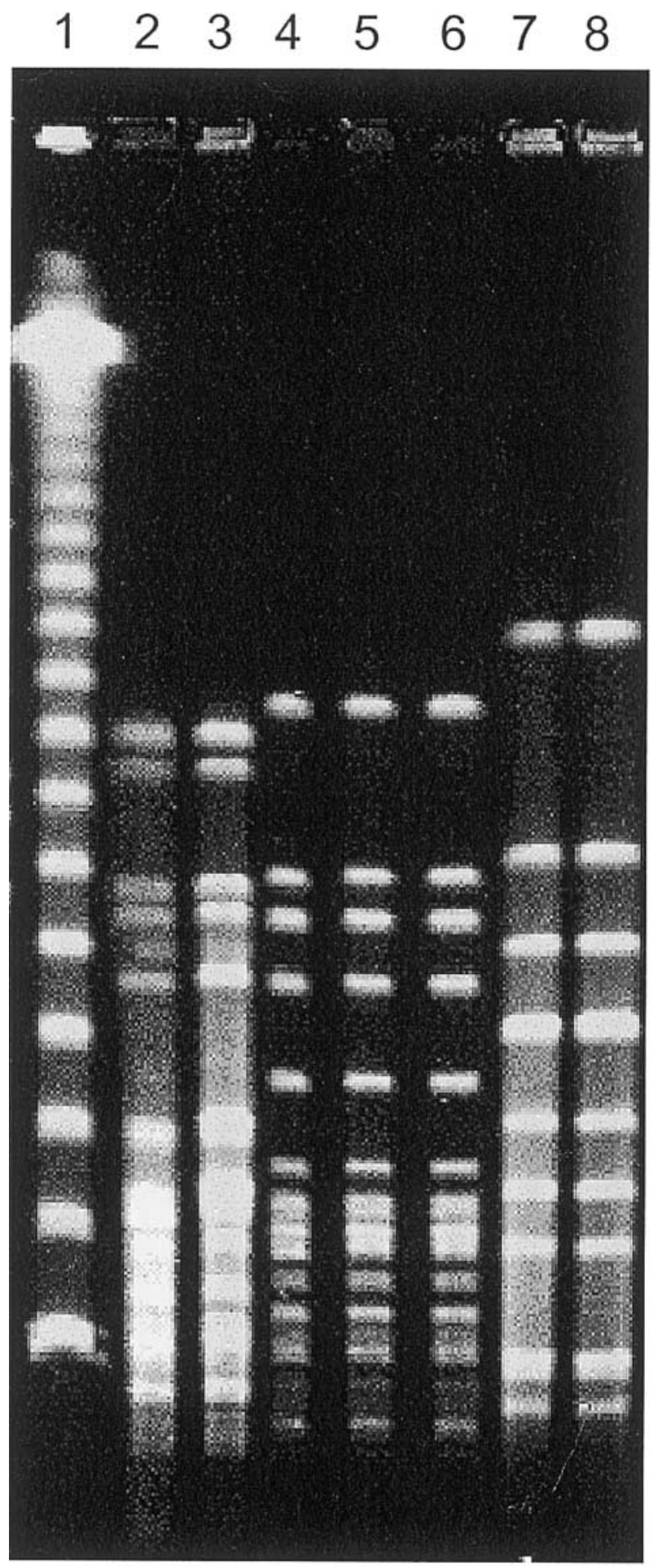

Fig. 1. PFGE of S. epidermidis and Str. oralis. Lane 1, DNA mol. wt marker (48.5-kb $\lambda$ ladder); 2, S. epidermidis PFGE type 1 from blood, day $7 ; 3, S$. epidermidis PFGE type 1 from mouth, days 4, 11, 21, 25 and nose, day $11 ; 4, S$. epidermidis PFGE type 2 from blood, days $11,29,70 ; 5$, S. epidermidis PFGE type 2 from blood, day $150 ; 6, S$. epidermidis PFGE type 2 from Hickman line tip, day 153; 7, Str. oralis from blood, day 7; 8, Str. oralis from mouth, day 4.

This scenario is similar to that described in 1994 when Lina et al. demonstrated that isolates of $S$. epidermidis from the throat and blood of a bacteraemic, neutropenic patient possessed identical PFGE patterns [7]. In 
this case, culture of the tip of the patient's Hickman line was negative.

In the present study, after the patient's first episode of bacteraemia, S. epidermidis PFGE type 2 colonised the Hickman line. After adherence to the line, subsequent production of biofilm would provide extra protection for these organisms from the effects of antimicrobial agents. S. epidermidis PFGE type 2 was responsible for a total of four episodes of bacteraemia before removal of the intravenous line.

In the present study, the antibiograms corresponded exactly with the PFGE types. In contrast, Lina et al. [8] demonstrated that isolates of $S$. epidermidis cultured from blood of a bone marrow transplant patient over a period of 5 months belonged to the same PFGE type, but possessed different antibiograms. This highlights the importance of molecular characterisation of strains.

In summary, this paper reports a case of bacteraemia caused by S. epidermidis and Str. oralis originating in the oral cavity, followed by infection by a second strain of $S$. epidermidis from a different site. Both the oral mucosa and central venous lines should be considered as potential sources of micro-organisms, including CNS, associated with bacteraemia in immunocompromised patients.

\section{References}

1. Das I, Philpott C, George RH. Central venous catheter-related septicaemia in paediatric cancer patients. J Hosp Infect 1997; 36: $67-76$.

2. Kloos WE, Bannerman TL. Update on clinical significance of coagulase-negative staphylococci. Clin Microbiol Rev 1994; 7: 117-140.

3. Bochud P-Y, Cometta A, Francioli P. Virulent infections caused by alpha-haemolytic streptococci in cancer patients and their management. Curr Opin Infect Dis 1997; 10: 422-430.

4. Steiner M, Villablanca J, Kersey J et al. Viridans streptococcal shock in bone marrow transplantation patients. Am J Hematol 1993; 42: 354-358.

5. Richard P, Del Valle GA, Moreau P et al. Viridans streptococcal bacteraemia in patients with neutropenia. Lancet 1995; 345: 1607-1609.

6. Stokes EJ, Ridgway GL. Clinical bacteriology, 5th edn. London, Arnold. 1980: 215.

7. Lina B, Forey F, Troncy J, Greenland T, Fleurette J, Etienne J. Oral source of Staphylococcus epidermidis septicemia in a neutropenic patient. Eur J Clin Microbiol Infect Dis 1994; 13: $773-775$.

8. Lina B, Forey F, Tigaud JD, Fleurette J. Chronic bacteraemia due to Staphylococcus epidermidis after bone marrow transplantation. J Med Microbiol 1995; 42: 156-160. 